\title{
An old foe still with us: Troubling issues with brucellosis
}

Turan BUZGAN ${ }^{1}$, İmran HASANOĞLU ${ }^{1}$

${ }^{1}$ Department of Infectious Diseases and Clinical Microbiology, Ankara Yildirim Beyazit University, School of Medicine, Ankara, Turkey.

SUMMARY

Although brucellosis is an old disease, it is still challenging for physicians. In this review, we discussed common pitfalls of brucellosis.

Key words: Brucellosis, endocarditis, neurobrucellosis, osteoarticular involvement

\section{INTRODUCTION}

Brucellosis is a bacterial zoonotic disease caused by Brucella species, which are Gram-negative, intracellular, and facultative anaerobe coccobacilli. Among Brucella bacteria that cause diseases in humans, Brucella melitensis is mainly found in sheep and goats, Brucella abortus is more common in cattle and mandarins, and Brucella suis is found in pigs. Brucella canis, which is found in dogs, is a rare cause of illness in humans. A majority of human brucellosis cases in endemic areas are due to $B$. melitensis; however, the disease caused by other species may be underestimated (1). Brucellosis is a rare zoonotic infection in developed countries, but it is still common in developing countries. In Turkey, the disease can be seen in all ages and gender, and the highest rate is seen between 15 and 35 years. Transmission from animals to humans occurs usually with direct contact of secretions of the infected animal with deteriorated skin, consumption of unpasteurized milk and dairy products, inhalation of infectious aerosols, or contact with conjunctiva. Contamination often occurs with consumption of unpasteurized dairy products in countries like Turkey where the disease is endemic (2).

A wide spectrum of clinical features of brucellosis can be seen in humans. It can affect any organ and mimic many infectious and noninfectious diseases. Brucella manages to avoid the host immune response and establish chronic infections (3). Since it can affect any organ, it may lead to hepatitis, orchitis, arthritis, endocarditis, and central nervous system infection (4). Of these, arthritis is the most common complication. Usually fever, fatigue, myalgia, and sweating are the initial symptoms $(2,5)$. As the disease progresses, loss of weight can be observed due to long-term anorexia.

Although brucellosis is an old disease, it is still challenging for physicians. In this review, we discussed the common pitfalls of brucellosis.

\section{Clinical features}

Brucellosis can be subclinical, acute, subacute, and chronic. It can present itself with nonspecific symptoms such as fever, night sweats, loss of appetite, weight loss, weakness, headache, and polyarthritis $(2,5-7)$. Brucellosis should be considered as a differential diagnosis for fevers of unknown origin. Debilitating conditions such as arthralgia, myalgia, and back pain affects around half of the patients. The incubation period is 2-3 weeks. Physical findings may vary depending on the duration of the disease. Systemic brucellosis sometimes can be complicated with meningitis, endocarditis, septic arthritis, and osteomyelitis. These complications are not extremely rare. A large meta-analysis reported the prevalence of endocarditis and neurobrucellosis as $1 \%$ and $4 \%$, respectively (6). Osteoarticular complications are the most common complications of brucellosis $(2,6,8)$.

Relapse can occur 2-3 months after the treatment and can be detected with serologic tests and blood culture positivity. Relapse rate is common in cases treated with a single antibiotic regimen and short-term treatment. 
Diagnosis

Laboratory findings include leukocytosis (especially focal complications), leukopenia, thrombocytopenia, and anemia. Also, a mild-to-moderate increase in erythrocyte sedimentation rate and C-reactive protein and moderate elevations in liver enzymes are observed. The diagnosis of brucellosis depends on the isolation of microorganisms from blood, bone marrow, or other tissues. However, positivity of the culture testing depends on many factors such as previous usage of antibiotics and ranges between 15.9 and $68 \%(2,5,9)$. Moreover, culture takes time and delays the diagnosis. Therefore, serologic testing becomes prominent with its rapid results. No well-defined cutoff levels exist for diagnosing brucellosis with serologic tests. The result can be assumed positive for titers equal to or greater than 1:80 in nonendemic regions and 1:160 in endemic regions $(2,10)$. However, a wide spectrum of manifestations of the disease complicates diagnosis. Low or borderline titers of agglutination test can lead to misdiagnosis or delayed diagnosis, especially in endemic regions. Therefore, clinicians must be careful if one of the differential diagnoses of the patient is brucellosis. A case study revealed that more than one third of patients had symptoms for 1-3 months prior to diagnosis and a delay in diagnosis of more than 30 days was associated with an increased risk of developing complicated brucellosis (9).

Brucel/a agglutination tests are crucial in the serological diagnosis of brucellosis. In routine practice, a positive Rose Bengal test result needs to be verified with standard agglutination test (SAT) and dilution tests (11). SAT is the most common method used for diagnosing brucellosis worldwide. Increased or positive SAT after a decreased or negative one can be an indicator of relapse or reinfection. Furthermore, it can be an anamnestic reaction due to antigenic similarity. 2- mercaptoethanol/Rivanol SAT or enzymelinked immunosorbent assay (ELISA) should be performed to distinguish the recurrence and anamnestic reaction. SAT becomes negative generally within 1 year after treatment. There is no need to control the post-treatment SAT. The disadvantages of SAT are possible false positive and false negative results, causing misinterpretation. False positive results can be seen with crossreactivity with other Gram-negative bacteria. Low titers or false negative results of agglutination tests can be a consequence of high antibody titers interfering with the formation of antigen-antibody complex, which is necessary for visualizing the agglutination. This is called prozone phenomenon, which always must be kept in mind for the diagnosis of brucellosis. It is usually seen in the presence of immunoglobulin $\lg (G)$-type antibodies. When the serum is diluted, this blockage is dissolved. Therefore, the serum should be diluted to at least 1/1280 titers, especially in endemic regions $(10,12)$. Coombs agglutination test (CAT) does not rule out this phenomenon. Prozone phenomenon can be seen in all forms of the disease. Other reasons forfalse negative results of agglutination tests are the presence of a blocking antibody, agammaglobulinemia, $B$. canis infections, undetectability in the early phases of the disease, and chronic brucellosis (13). Presence of the blocking antibody must be considered in all cases with a high suspicion of brucellosis, especially in endemic regions. Therefore, CAT, which neutralizes these blocking antibodies, becomes essential. Another serologic diagnostic test for brucellosis is immunocapture agglutination test (ICAT). This test is based on an immunocapture agglutination technique and detects nonagglutinating $\lg G$ and $\lg A$ antibodies, as well as agglutinating antibodies (11). Studies revealed that the specificity and sensitivity of CAT and ICAT methods were similar and, hence, both could be preferred to verify SAT $(14,15)$.

Brucella Coombs gel test is a new agglutination-based serologic test, which is practical, and yields results within $2 \mathrm{~h}$. Irvem et al reported that the gel test method showed excellent correlation with both CAT and ICAT tests (16). However, further studies are needed to recommend this test for the routine diagnosis of brucellosis.

Other serological tests used for diagnosing brucellosis are microagglutination test, ELISA, indirect fluorescent antibody test, and immunochromatographic lateral flow assay. ELISA helps in distinguishing between inactive and active brucellosis. It is a rapid test with high sensitivity and specificity, and measures Brucellaspecific lgM, lgG, and IgA. ELISA is the most appropriate technique for identifying acute cases. IgG antibodies become positive approximately 3 weeks after the onset of the disease. In general, titers of $\mathrm{lg} \mathrm{G}$ reach the highest levels in approximately $6-8$ weeks. After brucellosis is completely cured, lgG titers decline faster than $\operatorname{Ig} M$ titers. Therefore, detecting an increase in $\lg G$ and $\lg A$ titers using ELISA after the patient has recovered is a good indicator of relapse. ELISA is also an appropriate method for detecting antibodies in cerebrospinal fluid (CSF) in cases of neurobrucellosis (17).

Molecular diagnostic tests such as polymerase chain reaction $(P C R)$ are other options for diagnosing brucellosis, especially in neurobrucellosis and localized infections. PCR can be performed with whole blood, serum, and tissue. The sensitivity and specificity of PCR are reported to be higher than those of the culture $(18,19)$. 
However, PCR-based laboratory tests cannot be recommended for routine diagnosis of brucellosis because of its lack of standardization.

\section{Problematic issues regarding treatment and follow-up}

No one-size-fits-all treatment regimen exists for brucellosis. Therefore, treatment options need to be assessed for each patient based on the characteristics of the patient and course of the disease. Relapse or re-infection can be seen anytime throughout the patient's lifespan, and it is important to distinguish between these two conditions in terms of the approach to the patient. After effective treatment, patients often complain of similar symptoms and recurring illnesses. However, most of the cases have other causes. At this point, careful and detailed anamnesis and physical examination become essential. Laboratory tests should be performed considering other differential diagnoses. SAT with rivanol or 2- mercaptoethanol should be performed to identify the recurrence or anamnestic reaction. Relapse may occur after the end of the treatment, which is characterized by the recurrence of symptoms and an increase in SAT titer. Relapse is seen in about $5 \%$ of the patients after the treatment (2). The main reasons for relapse are inadequate treatment duration, presence of local complications requiring surgical treatment, and incompatibility of the patient with the treatment regimen.

Since the Brucella is located in the cell and phagolysosomes are acidic, antibiotics that pass into the cell and are effective in the acidic environment should be used. Monotherapy is not recommended because of its high recurrence rate. The most commonly used treatment regimen is doxycycline plus rifampin combination for 6 weeks (20). Triple-drug regimen should be preferred initially in special cases such as neurobrucellosis, endocarditis, and osteoarticular involvement. If fever persists despite proper antibiotic regimen, it is necessary to consider focal involvement or complication of brucellosis. Besides, patients must be evaluated for other possible fever etiologies in the case of ongoing fever.

\section{Musculoskeletal system involvement}

The most common complication of the disease is bone and joint involvement (20\%-85\%) (2,8). The most common clinical forms of osteoarticular involvement are arthritis, spondylitis, bursitis, tenosynovitis, and osteomyelitis. The most common regions are large or medium-sized peripheral joints, sacroiliac joints, and spinal region. Arthritis can be observed as monoarthritis or asymmetric peripheral oligoarthritis. Brucellacan sometimes cause destructive septic arthritis in the retained joint, and distinguishing brucellosis from radiographic tuberculous arthritis, especially in the coxofemoral joint and intervertebral joint, can be challenging (21). The recurrence rate is extremely low with triple-combination therapy in cases with osteoarticular involvement (22). Surgical intervention may be required for septic joint infections, wide paraspinal abscess, and some spondylitis cases. Paraspinal abscess treatment should be continued until radiological findings improve. The duration of treatment should be at least 3 months in cases with spondylitis (22).

\section{Neurobrucellosis}

Neurological involvement is $3 \%-5 \%$ in brucellosis (2). Since Brucella can affect all parts of the nervous system, the presentation of neurobrucellosis has a wide spectrum including meningitis, encephalitis, meningoencephalitis, myelitis, paresis, paresthesia, hemiplegia, meningovascular complications, parenchymatous dysfunction, peripheral neuropathy/radiculopathy, cerebral abscess, cerebellar abscess, Guillain-Barresyndrome, cranial nerve involvement, and myositis. The most frequent forms of neurological involvement are meningitis and meningoencephalitis. It may be difficult to distinguish neurobrucellosis from other forms of meningitis because it may be in the form of acute or chronic meningitis (23). Meningitis can be a complication that occurs in the late period of the disease as well as in early stages. CSF SAT for Brucella meningoencephalitis is valuable for diagnosis even if it is low titer positive (17).

Limited transmissibility of antibiotics to CSF is the distressing aspect of neurobrucellosis treatment. The treatment regimen of neurobrucellosis certainly must be able to cross the blood-brain barrier and achieve adequate CSF concentration. Since doxycycline is a lipophilic antibiotic, its CSF concentration is acceptable. Both doxycycline and rifampicin should be used in every combination due to their good passage to CSF. The concentration of streptomycin and gentamycin in CSF is therapeutic only when meninges are inflamed. From third-generation cephalosporins, The CSF passage of third-generation cephalosporins, such as cefotaxime, ceftriaxone, and ceftizoxime, is sufficient. Ceftriaxone + rifampicin + doxycycline is the best combination for treating neurobruce. The duration of treatment ranges from 6 weeks to 6 months (24). Improvement in the patient's clinic, normalization of CSF glucose, absence of leukocytes in CSF, and decrease in antibody titer in CSF are the criteria for the duration of treatment. Drainage should be performed in addition to antimicrobial treatment, if possible, in the brain abscess due to brucellosis. 


\section{Endocarditis}

Endocarditis is the most observed cardiovascular complication of Brucella. It usually involves the aortic valve and, less frequently, the mitral valve. Mycotic aneurysm, pericarditis, and myocarditis can be seen during the course of the disease (25). Both medical and surgical treatment may be required in patients with Brucella endocarditis. The triple-antibiotic regimen should be preferred. Doxycycline, rifampicin, streptomycin, co-trimoxazole, and ceftriaxone are good options (26).

\section{Brucellosis in pregnancy}

Brucellosis during pregnancy is associated with an increased risk of spontaneous abortion. Antimicrobial treatment at this point is extremely important for maternal and baby's health $(27,28)$. Doxycycline and streptomycin are not recommended during pregnancy because of their teratogenicity. Rifampicin, trimethoprim-sulfamethoxazole, and ceftriaxone are drugs preferred for brucellosis in pregnancy (29).

\section{CONCLUSIONS}

Brucellosis is a disease that causes mortality and morbidity. It is still endemic in Turkey and many other countries. It has gained significant attention owing to its multiple complications leading to a loss in the workforce. Clinicians must always keep brucellosis in mind in the differential diagnosis of fever of unknown origin.

\section{REFERENCES}

1. Doganay $M$, Mese EA. Bruselloz. In: Enfeksiyon Hastalıkları ve Mikrobiyolojisi. 3rd ed. Willke Topcu A, Soyletir G, Doganay M, Eds. Nobel Tip Kitabevleri, Istanbul, 2008: 897-909 (in Turkish).

2. Buzgan T, Karahocagil MK, Irmak H, Baran AI, Karsen $\mathrm{H}$, Evirgen $\mathrm{O}$, et al. Clinical manifestations and complications in 1028 cases of brucellosis: a retrospective evaluation and review of the literature. Int J Infect Dis IID Off Publ Int Soc Infect Dis. 2010 Jun;14(6): e469478.

3. Ahmed W, Zheng K, Liu Z-F. Establishment of Chronic Infection: Brucella's Stealth Strategy. Front Cell Infect Microbiol. 2016; 6:30.

4. de Figueiredo P, Ficht TA, Rice-Ficht A, Rossetti CA, Adams LG. Pathogenesis and immunobiology of brucellosis: review of Brucella-host interactions. Am J Pathol. 2015 Jun;185(6):1505-17.

5. Demiroğlu $Y Z$, Turunç $T$, Alişkan $H$, Colakoğlu $S$, Arslan $H$. Brucellosis: retrospective evaluation of the clinical, laboratory and epidemiological features of 151 cases. Mikrobiyol Bul. 2007 Oct:41(4):517-27.

6. Dean AS, Crump L, Greter H, Hattendorf J, Schelling E, Zinsstag J. Clinical manifestations of human brucellosis: a systematic review and meta-analysis. PLoS Negl Trop Dis. 2012;6(12): 1929.

7. Zribi $M$, Ammari L, Masmoudi A, Tiouiri H, Fendri C. [Clinical manifestations, complications and treatment of brucellosis: 45-patient study]. Pathol Biol (Paris). 2009 Jul;57(5):349-52.

8. Turan H, Serefhanoglu K, Karadeli E, Togan T, Arslan H. Osteoarticular involvement among 202 brucellosis cases identified in Central Anatolia region of Turkey. Intern Med Tokyo Jpn. 2011;50(5):421-8.
9. Colmenero JD, Reguera JM, Martos F, Sánchez-De-Mora D, Delgado $M$, Causse $M$, et al. Complications associated with Brucella melitensis infection: a study of 530 cases. Medicine (Baltimore). 1996 Jul;75(4):195-211.

10. Al Dahouk S, Tomaso H, Nöckler K, Neubauer H, Frangoulidis D. Laboratory-based diagnosis of brucellosis--a review of the literature. Part II: serological tests for brucellosis. Clin Lab. 2003;49(11-12):577-89.

11. Araj GF. Update on laboratory diagnosis of human brucellosis. Int J Antimicrob Agents. 2010 Nov;36 Suppl 1: S12-17.

12. Buzğan $T$, Karsen $H$, Karahocagil $M K$, Akdeniz $H$, Sunnetçioğlu M. [A case of brucellosis presenting as high titer negative result by standard tube agglutination test]. Mikrobiyol Bul. 2007 Jan;41(1):151-4.

13. Araj GF. Human brucellosis: a classical infectious disease with persistent diagnostic challenges. Clin Lab Sci J Am Soc Med Technol. 1999 Aug;12(4):207-12.

14. Orduña A, Almaraz A, Prado A, Gutierrez MP, Garcia-Pascual A, Dueñas $A$, et al. Evaluation of an immunocapture-agglutination test (Brucellacapt) for serodiagnosis of human brucellosis. J Clin Microbiol. 2000 Nov;38(11):4000-5.

15. Gómez MC, Nieto JA, Rosa C, Geijo P, Escribano MA, Muñoz A, et al. Evaluation of seven tests for diagnosis of human brucellosis in an area where the disease is endemic. Clin Vaccine Immunol CVI. 2008 Jun;15(6):1031-3.

16. İrvem $A$, Yücel FM, Aksaray $S$, Bor E. [Comparison of a new and rapid method, Brucella Coombs gel test with the other methods in the serological diagnosis of brucellosis]. Mikrobiyol Bul. 2015 Apr;49(2):181-7.

17. Guven T, Ugurlu K, Ergonul O, Celikbas AK, Gok SE, Comoglu S, et al. Neurobrucellosis: clinical and diagnostic features. Clin Infect Dis Off Publ Infect Dis Soc Am. 2013 May;56(10):1407-12.

18. Kaden R, Ferrari S, Alm E, Wahab T. A novel real-time PCR assay for specific detection of Brucella melitensis. BMC Infect Dis. 2017 Mar 24;17(1):230.

19. Yu WL, Nielsen K. Review of detection of Brucella spp. by polymerase chain reaction. Croat Med J. 2010 Aug;51(4):306-13.

20. Skalsky K, Yahav D, Bishara J, Pitlik S, Leibovici L, Paul M. Treatment of human brucellosis: systematic review and meta-analysis of randomised controlled trials. BMJ. 2008 Mar 29;336(7646):701-4.

21. al-Shahed MS, Sharif HS, Haddad MC, Aabed MY, Sammak BM, Mutairi MA. Imaging features of musculoskeletal brucellosis. Radiogr Rev Publ Radiol Soc N Am Inc. 1994 Mar;14(2):333-48.

22. Alp $E$, Doganay $M$. Current therapeutic strategy in spinal brucellosis. Int J Infect Dis IID Off Publ Int Soc Infect Dis. 2008 Nov;12(6):573-7.

23. Tarfarosh SFA, Manzoor M. Neurological Manifestations of Brucellosis in an Indian Population. Cureus. 2016 Jul 12;8(7):e684.

24. Pappas G, Akritidis N, Christou L. Treatment of neurobrucellosis: what is known and what remains to be answered. Expert Rev Anti Infect Ther. 2007 Dec;5(6):983-90.

25. Kula $S$, Erer $D$, Büyükates $M$, Tunaoglu $F S$, Olguntürk R, Ozdogan EM. Brucella endocarditis: case report and review of the literature. J Heart Valve Dis. 2001 Jul;10(4):486-8.

26. Koruk ST, Erdem H, Koruk I, Erbay A, Tezer-Tekce Y, Erbay AR, et al. Management of Brucella endocarditis: results of the Gulhane study. Int J Antimicrob Agents. 2012 Aug;40(2):145-50.

27. Khan MY, Mah MW, Memish ZA. Brucellosis in pregnant women. Clin Infect Dis Off Publ Infect Dis Soc Am. 2001 Apr 15;32(8):1172-7.

28. Kurdoglu M, Adali E, Kurdoglu Z, Karahocagil MK, Kolusari A, Yildizhan R, et al. Brucellosis in pregnancy: a 6-year clinical analysis. Arch Gynecol Obstet. 2010 Feb;281(2):201-6.

29. Mantur BG, Amarnath SK, Shinde RS. Review of clinical and laboratory features of human brucellosis. Indian J Med Microbiol. 2007 Jul;25(3):188-202. 